\title{
Palonosetrón comparado con ondansetrón y dexametasona en el control de náusea y vómito en el postoperatorio de colecistectomías laparoscópicas. Estudio de cohortes retrospectivas
}

\author{
Palonosetron compared with ondansetron and dexamethasone in \\ the control of nausea and vomiting in the postoperative period of \\ laparoscopic cholecystectomies. Retrospective cohort study
}

\author{
Joel Castellanos Chávez, ${ }^{*}$ María Elena Pinto Segura, ${ }^{\ddagger}$ Héctor Carrero Soto, ${ }^{\ddagger}$ \\ Cristhofer Abel Torres González, ${ }^{\ddagger}$ Ramsés Chacón*
}

Citar como: Castellanos CJ, Pinto SME, Carrero SH, Torres GCA, Chacón R. Palonosetrón comparado con ondansetrón y dexametasona en el control de náusea y vómito en el postoperatorio de colecistectomías laparoscópicas. Estudio de cohortes retrospectivas. Acta Med. 2021; 19 (1): 52-56. https://dx.doi.org/10.35366/98570

\section{Resumen}

La náusea y vómito postoperatorio (NVPO) en cirugía laparoscópica de colecistitis es frecuente. El uso de ondansetrón y dexametasona (GOD), y palonosetrón (GP) es muy prevalente. Objetivo: Comparar la eficacia en el control de la NVPO. Material y métodos: Realizamos un estudio comparativo de dos cohortes retrospectivas de 62 pacientes cada una con cirugía electiva de colecistectomía; uno con profilaxis con GOD y otro con GP. Se evaluó el riesgo de NVPO por la escala de Apfel. Las NVPO fueron estudiadas en las 24 horas postrecuperación de la anestesia. Resultados: No hubo diferencias en cuanto al sexo, valoración preanestésica y riesgo de NVPO entre los grupos. El 12.9\% (8/62) de los pacientes del grupo GOD presentó náuseas, contra ninguno del grupo GP $(p=0.003)$. Ningún paciente presentó vómitos postoperatorios $(p=1.00)$. En el grupo GOD seis requirieron rescate farmacológico $(9.6 \%)$, contra ninguno del GP $(p=0.014)$. No hubo efectos secundarios en ningún grupo. Conclusión: El uso de palonosetrón evidenció mejor control de la náusea, pero ambas estrategias profilácticas controlaron el vómito postoperatorio.

Palabras clave: Palonosetrón, ondansetrón, náusea, vómito, laparotomía, colecistectomía, náusea y vómito postoperatorio.

\section{Abstract}

Postoperative nausea and vomiting (PONV) in laparoscopic cholecystectomy surgery is common. The use of ondansetron and dexamethasone (GOD) and palonosetron (GP) is very prevalent. Objective: To compare the effectiveness in the control of the PONV. Material and methods: We conducted a comparative study of two retrospective cohorts of 62 patients each with elective cholecystectomy surgery; one with GOD prophylaxis and another with GP. The risk of PONV was assessed by the Apfel scale. The PONVs were studied in the 24 hours post-recovery of the anesthesia. Results: There were no differences regarding gender, pre-anesthetic assessment (ASA) and risk of PONV between the groups. 12.9\% (8/62) of the patients in the GOD group had nausea against those in the GP group $(p=0.003)$. No patient presented post-operative vomiting $(p=1.00)$. In the GOD group, six patients required pharmacological rescue $(9.6 \%)$ compared to none in the GP $(p=$ 0.014). There were no side effects in either group. Conclusion: The use of palonosetron evidenced better control of nausea, but both prophylactic strategies controlled post-operative vomiting.

Keywords: Palonosetron, ondansetron, nausea, vomiting, laparotomy, cholecystectomy, postoperative nausea and vomiting.

\footnotetext{
* Facultad Mexicana de Medicina, de la Universidad La Salle, Ciudad de México, México.

‡ Servicio de Anestesiología del Hospital Ángeles Clínica Londres. Ciudad de México, México.
}

\section{Correspondencia:}

Joel Castellanos Chávez

Correo electrónico: medcastellanos@gmail.com

Aceptado: 18-05-2020.

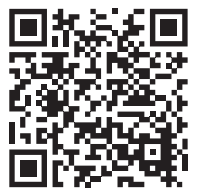




\section{INTRODUCCIÓN}

Las náuseas y vómitos postoperatorios (NVPO) son problemas frecuentes en la anestesia laparoscópica. ${ }^{1}$ Las NVPO no sólo disminuyen el grado de satisfacción del paciente, también aumentan los costos hospitalarios, retrasando altas y exigiendo ingresos no esperados. ${ }^{2}$ Las NVPO pueden prolongar el tiempo de estadía en las unidades de cuidados postanestésicos (UCPA) y aumentar los costos de la cirugía. ${ }^{3,4}$

Los factores de riesgo encuentran su aplicación clínica en las escalas pronósticas del riesgo, que permiten clasificar a los pacientes según su riesgo de presentar NVPO y decidir si prevenir o tratar. En la década de los 90 se desarrolló la escala simplificada de Apfel (Tabla 1), la cual resultó ser igualmente eficaz que sus predecesoras, y al ser simplificada y darle el mismo peso a cada factor de riesgo, es fácilmente aplicable y es la más utilizada en la actualidad. ${ }^{5}$

A pesar de existir lineamientos generales y acciones preventivas no medicamentosas profilácticas para prevenir su aparición, ${ }^{4,6}$ en ocasiones es necesario recurrir a fármacos cuando el riesgo de su presentación es moderado, como es en las cirugías laparoscópicas. ${ }^{7}$ En estas condiciones, la recomendación es el uso de monoterapia o biterapia anti-NVPO. ${ }^{8,9}$

En la actualidad, los fármacos más recomendados para la cirugía de colecistectomía laparoscópica (CCL) han sido los antagonistas de los receptores de serotonina (5-HT3) o setrones. ${ }^{10-12}$ El más usado ha sido el ondansetrón, un inhibidor competitivo del receptor del $5-\mathrm{HT} 3^{10}$ con buenos resultados, pero no exento de reacciones adversas, como son: cefalea, elevación de las enzimas hepáticas sin repercusión clínica y estreñimiento; de ahí su frecuente asociación con dexametasona. ${ }^{13}$ En un intento de control con monoterapia se ha propuesto el uso del palonosetrón. ${ }^{14,15}$
El palonosetrón es un antagonista de mayor afinidad al receptor 5-HT3 y, por tanto, una vida media más larga. ${ }^{16}$ Por otro lado, a diferencia de los setrones de primera generación, el palonosetrón no prolonga el intervalo QT. ${ }^{16}$

A pesar de su aprobación para su uso, existen escasos reportes de la experiencia en el control de la NVPO en CCL en pacientes mexicanos. Por lo anterior, el objetivo de este estudio fue comparar la efectividad de ondansetrón y dexametasona contra palonosetrón en el control de NVPO en CCL dentro de las primeras 24 horas postoperatorias y de estancia hospitalaria.

\section{MATERIAL Y MÉTODOS}

Realizamos un estudio de cohortes retrospectivas de pacientes mayores de 18 años, atendidos en el Hospital Ángeles Clínica Londres por colecistitis aguda y sometidos a cirugía de colecistectomía vía laparoscópica. Todos con un riesgo quirúrgico ASA I o II, quienes recibieron anestesia general balanceada y administración de ondansetrón y dexametasona o palonosetrón. La decisión del uso de estos antieméticos fue del anestesiólogo responsable de la anestesia de cada caso. Durante el periodo de enero de 2017 a diciembre de 2018 se realizaron 938 CCL.

Debido a que deseábamos contestar la hipótesis de una diferencia no mayor de $10 \%$ de NVPO para aceptar un efecto profiláctico, y un riesgo de cometer un error tipo I de menos del 5\%, se estimó un tamaño mínimo de 62 pacientes por grupo. Los grupos fueron completados por un muestreo a conveniencia, una vez comprobado en cada expediente clínico la administración de estos fármacos y la evaluación de su efecto sobre NVPO.

En los casos con manejo con ondansetrón (GDO); el protocolo del hospital consistía en administrar dexametasona a 8 mg vía intravenosa inmediatamente después de la

Tabla 1: Escala de Apfel y estimulación del riesgo.

Escala de Apfel

Factores de riesgo

Ninguno

Sexo femenino

No fumar

Historia de NVPO o cinetosis

Opioides postoperatorios

Suma
Estimulación del riesgo

Puntos

Probabilidad de riesgo NVPO \%

NVPO = náusea y vómito postoperatorio. 
Tabla 2: Características demográficas de los pacientes sometidos a colecistectomía por laparoscopia que recibieron ondansetrón y dexametasona contra sólo palonosetrón.

\begin{tabular}{|c|c|c|c|c|}
\hline & $\begin{array}{c}\text { Grupo GOD }(n=62) \\
n(\%)\end{array}$ & $\begin{array}{c}\text { Grupo GP }(n=62) \\
n(\%)\end{array}$ & $p$ & $\%$ diferencia $\left(\mathrm{IC}_{95 \%}\right)$ \\
\hline Sexo & & & 1.00 & 3.5 (-1 a 1.5) \\
\hline Masculino & $20(32.2)$ & $21(33.9)$ & & \\
\hline Femenino & $42(67.8)$ & $41(66.1)$ & & \\
\hline ASA & & & 0.068 & 11.3 (-6 a 28.7) \\
\hline 1 & $31(50.0)$ & $24(38.7)$ & & \\
\hline II & $31(50.0)$ & 38 (61.3) & & \\
\hline Escala riesgo Apfel & & & 0.138 & $3.2(-20.2$ a 13.8$)$ \\
\hline Bajo & $22(35.4)$ & $24(38.7)$ & & \\
\hline Moderado & $40(64.5)$ & 38 (61.3) & & \\
\hline
\end{tabular}

inducción anestésica y el ondansetrón a dosis de 8 mg vía intravenosa al final del procedimiento quirúrgico.

Para el grupo de palonosetrón (GP), éste fue administrado a dosis de $0.075 \mathrm{mg}$ vía intravenosa inmediatamente antes de la inducción y como dosis única. ${ }^{17}$

En cada persona se obtuvo información de su sexo, su ASA y la evaluación preanestésica del riesgo de NVPO (escala de Apfel). ${ }^{18}$ Del expediente tanto médico como de enfermería se obtuvo información sobre la presencia de náusea y el registro de vómitos. Éstos fueron resumidos en periodos de tiempo preestablecidos: de cero a dos horas postoperatoria, y de dos a 24 horas postoperatorias. Asimismo, se revisó si el paciente recibió medicación de rescate por presencia de náuseas o vómitos durante el periodo postoperatorio.

El proyecto fue aprobado por el Comité de Investigación y Ética del Hospital Ángeles Clínica Londres con el folio 007 del 2018. Al ser un estudio observacional, no requirió de carta de consentimiento informado y sólo se respetó el anonimato y confidencialidad de los datos.

Análisis estadístico. Las mediciones fueron resumidas en frecuencias simples y relativas en porcentajes por grupos. Para contrastar las diferencias, se utilizó prueba no paramétrica de $\chi^{2}$ con corrección de Yates entre las proporciones entre grupos. El análisis se realizó con el programa estadístico SPSS versión 22. Las diferencias se presentan con sus intervalos de confianza al 95\%. Se consideró un nivel de significancia estadística con un valor de $p<0.05$.

\section{RESULTADOS}

En la Tabla 2 presentamos la comparación de los grupos en sus características demográficas. Como se muestra, hubo una mayor frecuencia de mujeres en ambos grupos, la razón general en toda la muestra fue de 1.9 mujeres por un hombre. La distribución del riesgo anestésico también fue semejante entre grupos con un $11.3 \%$ ( $p=0.068$ ) más de casos ASA II en el GP. Por último, en la evaluación del riesgo de náusea y vómito (Apfel) encontramos un 3.2\% más de pacientes con categoría moderada en el GOD con respecto al GP ( $p=0.138)$.

Durante el proceso anestésico, ningún paciente tuvo alteraciones en sus signos vitales que indicaran manejo farmacológico (datos no presentados). Asimismo, todos los procedimientos estudiados fueron exitosos y sin complicaciones transquirúrgicas.

En la evolución de la náusea y vómito postanestésico (Tabla 3) se observó un control de ambas complicaciones durante las 24 horas de vigilancia en todos los casos manejados con palonosetrón (eficacia del 100\%). Por otro lado, en ocho pacientes del GDO, todos con riesgo moderado (Apfel), se informó sobre la presencia de náuseas en las primeras dos horas del ingreso a recuperación, el cual se resolvió con uso de medicamentos (metoclopramida) en seis. Ningún caso de este grupo presentó vómitos. Tampoco se documentó presencia de alteraciones del ritmo cardiaco.

\section{DISCUSIÓN}

La NVPO en cirugía laparoscópica de colecistectomía ha sido reportada en diversos estudios previos, de ahí la recomendación del uso profiláctico de medicamentos antieméticos. ${ }^{1,19}$ En este estudio valoramos la experiencia en efectividad del control de estas complicaciones con dos esquemas terapéuticos ampliamente usados en nuestra unidad médica: ondansetrón y palonosetrón. Nuestra 
hipótesis sobre su eficacia en el control de la NVPO era una eficiencia semejante alta en ambos esquemas. Nuestros datos mostraron una baja incidencia de estas dos complicaciones en las siguientes 24 horas después de terminada la anestesia. El uso de palonosetrón fue efectivo en todos los casos y el ondansetrón en más del $80 \%$ de los participantes.

Una revisión sistemática reciente dio evidencia a favor del uso de palonosetrón en el control postoperatorio del NVPO en cirugía; sin embargo, el análisis particular en procedimientos laparoscópicos no fue evaluado. ${ }^{20} \mathrm{Su}$ mayor eficacia en cirugía laparoscópica como la de nuestros pacientes ha sido menos estudiada. Dos estudios recientes, ${ }^{11,21}$ ambos ensayos clínicos controlados aleatorizados, comparando estos dos esquemas informaron igualmente una efectividad semejante. En el estudio de Laha, ${ }^{21}$ los pacientes tanto en el grupo tratado con palonosetrón como el de ondansetrón tuvieron una eficiencia completa (ausencia de NVPO) del 32.7 y $28 \%$, con una muestra de 40 pacientes por grupo. Esto contrasta con nuestra eficiencia completa en el 100\% de nuestro GP y del $90 \%$ (57/62) del GDO. Dos explicaciones surgen, la primera es el instrumento para medir la náuseas, ellos usaron una escala análoga visual de sensación de náusea y nosotros la información documentada. Al ser su estudio prospectivo, es más factible su evaluación y más objetiva, y no el sesgo de registro potencialmente presentado en nuestro estudio. Por otro lado, en la presencia de vómitos, datos más objetivos, tanto en su estudio como en el nuestro, no se reportaron en ningún paciente. Los autores concluyeron un control del vómito igualmente eficaz con ambos esquemas. Es importante señalar las mismas características de edad en nuestro estudio al de éste.

Por otro lado, otra publicación más reciente ${ }^{11}$ en mujeres con mayor riesgo de NVPO (> 60 años), sometidas también a colecistectomía selectiva por laparoscopia y con un esquema de dosis única de palonosetrón pero múltiple con ondansetrón (inmediata, 6 y 12 h), mostró mejor control de la náusea y vómito con el primero (57 y 55\% respectivamente). Sin embargo, también la frecuencia de náusea fue en general más alta, posiblemente por la edad de los pacientes. El vómito fue controlado igual por ambos esquemas.

Como en los estudios anteriores, nosotros tampoco logramos documentar efectos secundarios severos en ningún grupo.

Nuestra investigación, por su naturaleza observacional y retrospectiva, presenta desventajas sobre los estudios antes comentados. La selección de los medicamentos no fue aleatoria, pero los pacientes presentaron riesgo bajo y moderado. Es factible que la diferencia principal en el control de la NVPO se presentara en los casos con riesgo moderado al mismo y por el número de pacientes un análisis estratificado no fue posible. Como se comentó previamente, la información de nuestro estudio fue de fuentes secundarias $y$, por tanto, la confiabilidad puede ser baja; sin embargo, resultados más objetivos como la presencia de vómitos son más confiables. Además, consideramos que el sesgo no fue diferencial a los grupos, por lo que el resultado puede ser más aproximado a la realidad.

Por último, es importante difundir en nuestra comunidad la experiencia en centros con poblaciones mexicanas para explorar posibles respuestas diferentes a las informadas en el resto del mundo.

\section{CONCLUSIÓN}

En este estudio encontramos que el manejo profiláctico anti NVPO con palonosetrón dosis única puede ser más efectivo en su control con respecto al uso de ondansetrón. Sin embargo, el control por ambos esquemas es adecuado para su indicación.

\begin{tabular}{|c|c|c|c|c|}
\hline & Grupo GOD $(n=62)$ & $\%\left(\mathrm{IC}_{95 \%}\right)$ & Grupo GP $(n=62)$ & $\mathrm{p}$ \\
\hline Náusea & & & & 0.003 \\
\hline $0 \mathrm{a} 2 \mathrm{~h}$ & 8 & 12.9 (6 a 24) & 0 & \\
\hline 3 a $24 \mathrm{~h}$ & 0 & 0 & 0 & \\
\hline Vómito & & & & 1.00 \\
\hline $0 \mathrm{a} 2 \mathrm{~h}$ & 0 & 0 & 0 & \\
\hline 3 a $24 \mathrm{~h}$ & 0 & 0 & 0 & \\
\hline Rescate & 6 & 9.6 (4 a 20) & 0 & 0.014 \\
\hline
\end{tabular}




\section{REFERENCIAS}

1. Turkistani A, Abdullah K, Manaa E, Delvi B, Khairy G, Abdulghani B et al. Effect of fluid preloading on postoperative nausea and vomiting following laparoscopic cholecystectomy. Saudi J Anaesth. 2009; 3 (2): 48-52.

2. Hill RP, Lubarsky DA, Phillips-Bute B, Fortney JT, Creed MR, Glass PS et al. Cost-effectiveness of prophylactic antiemetic therapy with ondansetron, droperidol, or placebo. Anesthesiology. 2000; 92 (4): 958-967.

3. Tramèr MR. A rational approach to the control of postoperative nausea and vomiting: evidence from systematic reviews. Part II. Recommendations for prevention and treatment, and research agenda. Acta Anaesthesiol Scand. 2001; 45 (1): 14-19.

4. Gan TJ, Diemunsch P, Habib AS, Kovac A, Kranke P, Meyer TA et al. Consensus guidelines for the management of postoperative nausea and vomiting. Anesth Analg. 2014; 118 (1): 85-113.

5. Apfel CC, Laara E, Koivuranta M, Greim CA, Roewer N. A simplified risk score for predicting postoperative nausea and vomiting: conclusions from cross-validations between two centers. Anesthesiology. 1999; 91 (3): 693-700.

6. Gómez-Arnau JI, Aguilar JL, Bovaira P, Bustos F, de Andrés J, de la Pinta JC, et al. Recomendaciones de prevención y tratamiento de las náuseas y vómitos postoperatorios y/o asociados a las infusiones de opioides. Rev Esp Anestesiol Reanim. 2010; 57 (8): 508-524.

7. Arslan M, Ciçek R, Kalender HU, Yilmaz H. Preventing postoperative nausea and vomiting after laparoscopic cholecystectomy: a prospective, randomized, double-blind study. Curr Ther Res Clin Exp. $2011 ; 72(1): 1-12$.

8. Lagos AC, Quezada ES. Profilaxis y tratamiento de las náuseas y vómito postoperatorios. Rev Chil Anest. 2009; 38 (1): 24-33.

9. Scholz J. Essential drugs in anesthetic practice. Antiemetics. Anesthetic pharmacology. 2nd. United Kingdom: Cambridge University Press; 2011. pp. 855-873.

10. Thompson AJ, Lummis SC. The $5-\mathrm{HT} 3$ receptor as a therapeutic target. Expert Opin Ther Targets. 2007; 11 (4): 527-540.

11. Carvalho Braga EL, Figueiredo NV, Barrucand L, Cavalcanti IL. Use of palonosetron and ondansetron in the prophylaxis of postoperative nausea and vomiting in women 60 years of age or older undergoing laparoscopic cholecystectomy: A randomised double-blind study. Eur J Anaesthesiol. 2019; 36 (3): 241-242. doi: 10.1097/ EJA.0000000000000932.

12. Niesler B, Walstab J, Combrink S, Möller D, Kapeller J, Rietdorf J et al. Characterization of the novel human serotonin receptor subunits 5-HT3C,5-HT3D, and 5-HT3E. Mol Pharmacol. 2007; 72 (1): 8-17.

13. Henzi I, Walder B, Tramer MR. Dexamethasone for the prevention of postoperative nausea and vomiting: a quantitative systematic review. Anesth Analg. 2000; 90 (1): 186-194.

14. Rojas C, Stathis M, Thomas AG, Massuda EB, Alt J, Zhang J et al. Palonosetron exhibits unique molecular interactions with the 5-HT3 receptor. Anesth Analg. 2008; 107 (2): 469-478.

15. Wallenborn J, Kranke P. Palonosetron hydrochloride in the prevention and treatment of postoperative nausea and vomiting. Clinical Medicine Insights: Therapeutics. 2010; 2: 387-399.

16. Rojas C, Thomas AG, Alt J, Stathis M, Zhang J, Rubenstein EB et al. Palonosetron triggers 5-HT(3) receptor internalization and causes prolonged inhibition of receptor function. Eur J Pharmacol. 2010; 626 (2-3): 193-199.

17. Kovac AL, Eberhart L, Kotarski J, Clerici G, Apfel C; Palonosetron 04-07 Study Group. A randomized, double-blind study to evaluate the efficacy and safety of three different doses of palonosetron versus placebo in preventing postoperative nausea and vomiting over a 72-hour period. Anesth Analg. 2008; 107 (2): 439-444.

18. Pleuvry B. Physiology and pharmacology of nausea and vomiting. Anaesth Intens Care Med. 2006; 7 (12): 473-477.

19. Park SK, Cho EJ. A randomized, double-blind trial of palonosetron compared with ondansetron in preventing postoperative nausea and vomiting after gynaecological laparoscopic surgery. J Int Med Res. 2011; 39 (2): 399-407.

20. Xiong C, Liu G, Ma R, Xue J, Wu A. Efficacy of palonosetron for preventing postoperative nausea and vomiting: a systematic review and meta-analysis. Can J Anaesth. 2015; 62 (12): 1268-1278.

21. Laha B, Hazra A, Mallick S. Evaluation of antiemetic effect of intravenous palonosetron versus intravenous ondansetron in laparoscopic cholecystectomy: a randomized controlled trial. Indian J Pharmacol. 2013; 45 (1): 24-29. 